\title{
Development and Application of a Quantitative PCR Detection Method to Quantify Venturia oleaginea in Asymptomatic Olive (Olea europaea) Leaves
}

\author{
Silvia Scibetta, ${ }^{1}$ Giovanni E. Agosteo, ${ }^{1}$ Ahmed Abdelfattah, ${ }^{2}$ Maria G. Li Destri Nicosia, ${ }^{1}$ Santa O. Cacciola, ${ }^{3}$ \\ and Leonardo Schena ${ }^{1, \dagger}$
}

\author{
${ }^{1}$ Dipartimento di Agraria, Università Mediterranea, Reggio Calabria, Italy \\ ${ }^{2}$ Department of Ecology, Environment and Plant Sciences, Stockholm University, Stockholm, Sweden \\ ${ }^{3}$ Dipartimento di Agricoltura, Alimentazione e Ambiente, Università degli Studi, Catania, Italy \\ Accepted for publication 15 October 2019.
}

\begin{abstract}
Olive leaf spot (OLS), caused by Venturia oleaginea, is one of the most common and serious diseases of olive trees in the Mediterranean region. Understanding the pathogen life cycle is important for the development of effective control strategies. Current knowledge is incomplete owing to a lack of effective detection methods. It is extremely difficult to culture $V$. oleaginea in vitro, so primers were designed to amplify and sequence the internal transcribed spacer ITS1-5.8S-ITS2 region of the fungus directly from infected olive leaves. Sanger sequencing indicated a unique ITS region present in the European strains screened, confirming the appropriateness of the target region for developing a quantitative PCR (qPCR) assay. Furthermore, high-throughput sequencing of the same region excluded the presence of other Venturia species in the olive phyllosphere. The qPCR assay proved very specific and sensitive, enabling the detection of approximately 26 copies of target DNA. The analysis of symptomless
\end{abstract}

ABSTRACT leaves during early stages of the epidemic from the end of winter through spring revealed a similar quantity of pathogen DNA regardless of the leaf growth stage. In contrast, the pathogen titer changed significantly during the season. Data indicated that leaf infections start earlier than expected over the season and very young leaves are as susceptible as adult leaves. These findings have important practical implications and suggest the need for improved scheduling of fungicide treatments. The qPCR assay represents a valuable tool providing quantitative results and enables detection of $V$. oleaginea in all olive organs, including those in which OLS cannot be studied using previously available methods.

Keywords: amplicon metagenomics, disease control and pest management, latent infections, molecular detection, mycology, olive leaf spot, olive scab, peacock's eye disease, quantitative PCR, techniques
Olive leaf spot (OLS), also known as olive scab, peacock leaf spot, or peacock's eye disease, is caused by the fungus Venturia oleaginea (Castagne) Rossman \& Crous [synonyms Spilocaea oleagina (Cast.) Hugh and Fusicladium oleagineum (Castagne) Ritschel \& U. Braun] and is one of the most common and serious diseases of olive groves in the Mediterranean region (Agosteo and Schena 2011). Infections occur mainly on leaves, but other organs, including shoots, leaf stalks, fruit pedicles, fruits, and inflorescences, can also be infected. On susceptible cultivars, severe defoliation may occur, resulting in a loss of plant vigor, death of shoots, and a reduction in the numbers of flower buds and, consequently, in fruit production (Agosteo and Schena 2011).

The pathogen can infect olive tissues over a wide range of temperatures $\left(5\right.$ to $25^{\circ} \mathrm{C}$ ) provided that prolonged conditions of leaf wetness occur. At least $24 \mathrm{~h}$ of leaf wetness was found to be necessary for infections at the optimal temperature of $20^{\circ} \mathrm{C}$ (Viruega and Trapero-Casas 2002). Once the pathogen penetrates the host, it remains restricted to the outer cuticle layer of the epidermal cell wall, where the mycelium grows parallel to the leaf surface to form round,

${ }^{\dagger}$ Corresponding author: L. Schena; 1schena@unirc.it

Funding: This work was supported by an Italian Ministry of Education, University and Research (MIUR) grant ("Modelli sostenibili e nuove tecnologie per la valorizzazione delle olive e dell'olio extravergine di oliva prodotto in CalabriaPON Ricerca e competitivita 2007-2013," PON03PE_00090_02).

G. E. Agosteo and A. Abdelfattah contributed equally to this work.

*The $\boldsymbol{e}$-Xtra logo stands for "electronic extra" and indicates that one supplementary table is published online.

The author(s) declare no conflict of interest.

(c) 2020 The American Phytopathological Society flat, monolayered, immersed colonies (Graniti 1993; Lanza et al. 2017). Colonization is followed by the appearance of typical symptoms and by the eruption of conidia formed on short bulbous single-celled conidiophores. The duration of the incubation period depends on local environmental conditions, leaf age, and varietal susceptibility and may vary from a minimum of 10 days up to several months. In particular, when infections occur just before cold (winter) or dry and hot (summer) conditions, symptom development and sporulation may occur after a long period of latency (Viruega et al. 2013). The duration of summer and winter latency periods depends on local environmental conditions, although summer latency prevails in most Mediterranean olive-growing areas as a result of typical dry-hot summers and mild-wet winters. In any case, latent infections are extremely important because they represent the main overwintering and oversummering strategy of the pathogen, and understanding this stage is essential for the development of effective control strategies (Graniti 1993; Viruega et al. 2013).

Chemical control is very important in areas with a high or medium risk of infection and on susceptible cultivars because nontreated trees may decline rapidly in productivity. Copper-based fungicides applied during active sporulation and infection periods (generally autumn and late-winter/spring) are the most frequently adopted. The copper ion can enter the cuticle through wounds caused by erupting conidia and cause premature leaf drop, but it controls the disease. Conidia produced on fallen leaves are of no practical importance for new infections, owing to their low dispersal ability (Agosteo and Schena 2011). Cupric treatments should be avoided where there is a very high incidence of scab because they may induce an excessive, early loss of leaves caused by $\mathrm{C}_{\mathrm{U}^{++}}$ion phytotoxicity. Thus, accurate monitoring of the pathogen during latent and symptomatic phases is essential to determine the timing of fungicide applications and the number of treatments and appropriate chemicals to apply (Agosteo and Schena 2011). 
However, the monitoring of latent infections is very complex because the diagnosis of the disease is based mainly on symptoms. In fact, $V$. oleaginea is very difficult, if not impossible, to isolate on nutrient media in vitro (González-Lamothe et al. 2002; Wilson and Miller 1949). A simple method to visualize the disease (if symptomless) is based on the observation of round black spots after the leaves have been immersed in sodium or potassium hydroxide solution (Loprieno and Tenerini 1959). This method has been widely applied to study the life cycle of $V$. oleaginea and to determine the duration of the latent phase. However, important epidemiological aspects remain controversial because the Loprieno and Tenerini method enables the identification of infected leaves only if the pathogen is at an advanced stage of colonization; this method is ineffective during the early stages of infection. Furthermore, it does not provide accurate quantification and can be utilized only for adult leaves because young, tender leaves are damaged by sodium hydroxide during the immersion process.

Thus, a specific and sensitive molecular method is desirable to detect and quantify the pathogen over the entire infection process and provide a tool to accurately study its life cycle (Schena et al. 2004). The early infection phases on immature, expanding leaves are particularly important because they are critical to plant productivity; currently, knowledge on the infection at this stage is incomplete and controversial. Therefore, this study aimed to develop and apply a quantitative PCR (qPCR) method based on TaqMan chemistry to detect $V$. oleaginea in symptomless olive leaves at various growth stages. The development and validation of the method was challenging considering the difficulty in obtaining pure cultures of $V$. oleaginea and related species (GonzálezLamothe et al. 2002). To avoid this shortcoming, the whole internal transcribed spacer ITS1-5.8S-ITS2 sequence of the pathogen was directly amplified from naturally infected olive leaves and utilized as a target to design two specific primers and a probe.

\section{MATERIALS AND METHODS}

DNA extraction from olive leaves. All DNA extractions were performed from lyophilized leaf tissues using a modified version of the CTAB protocol (Doyle and Doyle 1987). A sample of $40 \mathrm{mg}$ of lyophilized tissue was blended at $25 \mathrm{~Hz}$ for two 1-min periods in a Mixer Mill homogenizer (Retsch Technology GmbH, Haan, Germany) containing 1:2 (wt/vol) CTAB lysis buffer and $0.3 \mathrm{~g}$ of glass beads $(0.1$ to $3 \mathrm{~mm} \emptyset$ ). The buffer contained $100 \mathrm{mM}$ of Tris- $\mathrm{HCl}(\mathrm{pH} \mathrm{8}), 1.4 \mathrm{M}$ of $\mathrm{NaCl}, 20 \mathrm{mM}$ of EDTA (pH 8), 2\% $\mathrm{CTAB}$, and $2 \%$ polyvinylpolypyrrolidone (Sigma-Aldrich, St. Louis, MO). The homogenate was incubated at $65^{\circ} \mathrm{C}$ for $30 \mathrm{~min}$ and the supernatant was extracted with chloroform-isoamyl alcohol $24: 1(\mathrm{vol} / \mathrm{vol})\left(-20^{\circ} \mathrm{C}\right)$ and precipitated with a 0.2 volume of $5 \mathrm{M}$ $\mathrm{NaCl}$ and $100 \%$ cold $\left(-20^{\circ} \mathrm{C}\right)$ ethanol $(2.5 \mathrm{vol} / \mathrm{vol})$. The pellet was washed with $70 \%$ (vol/vol) ethanol and dried in a heating block at $37^{\circ} \mathrm{C}$ for $5 \mathrm{~min}$. DNA was suspended in $50 \mu \mathrm{l}$ of nuclease-free water and the concentration and quality (ratios A260/A280 and A260/ A230) was evaluated with a NanoDrop 1000 spectrophotometer (NanoDrop Technologies, Wilmington, DE) and by electrophoresis using a $1.5 \%$ agarose gel in TBE buffer $(90 \mathrm{mM}$ of Tris, $90 \mathrm{mM}$ of boric acid, and $1 \mathrm{mM}$ of EDTA).

Amplification and Sanger sequencing of the ITS region of $\boldsymbol{V}$. oleaginea. New primers were designed to amplify the ITS regions of $V$. oleaginea from infected olive samples. GenBank available ITS sequences of $V$. oleaginea were aligned with a representative panel of sequences from related fungal species (Supplementary Table S1). Alignments were checked manually to identify appropriate target regions for the amplification of the whole ITS1-5.8S-ITS2 region from $V$. oleaginea and closely related species. Two primers targeting the final portion of the $18 \mathrm{~S}$ gene (Ve1F GAACCTGCGGAAGGATCA) and the boundary between ITS2 and the 28S gene (Ve2R ATCCGAGGCCAACCTTGTAG) were designed using PRIMER 3 software with default parameters.
Primers were utilized to amplify target DNA from leaf samples showing typical symptoms of OLS collected in Italy, Greece, France, and Spain (Table 1). Amplifications were performed according to the protocol for GoTaq Flexi DNA Polymerase (Promega Corporation, Madison, WI). A total volume of $50 \mu \mathrm{l}$ contained $10 \mu \mathrm{l}$ of $5 \times$ GoTaq Flexi Buffer, $1.5 \mathrm{mM}$ of $\mathrm{MgCl}_{2}, 0.3 \mu \mathrm{M}$ of each primer, $0.2 \mathrm{mM}$ of dNTPs, $1.25 \mathrm{U}$ of GoTaq DNA Polymerase, and $2 \mu \mathrm{l}(20 \mathrm{ng})$ of extracted DNA. PCR conditions consisted of an initial step at $95^{\circ} \mathrm{C}$ for $2 \mathrm{~min}$, followed by 35 cycles at $95^{\circ} \mathrm{C}$ for $30 \mathrm{~s}$, annealing at $55^{\circ} \mathrm{C}$ for $30 \mathrm{~s}$, and $72^{\circ} \mathrm{C}$ for $40 \mathrm{~s}$. A final extension at $72^{\circ} \mathrm{C}$ for $5 \mathrm{~min}$ was included. All reactions were incubated in a Mastercycler gradient thermocycler (Eppendorf, Hamburg, Germany). The PCR fragments were visualized on $1.5 \%$ agarose gels and bidirectionally sequenced with the same primers, using the BigDye Terminator v3.1 Cycle Sequencing Kit and an ABI3500 automated sequencer (Applied Biosystems, Foster City, CA).

High-throughput sequencing. $V$. oleaginea and related species associated with the olive phyllosphere were preliminarily investigated using a metabarcoding approach with the primers Ve1F and Ve2R. Analyses were conducted with four biological samples collected in four Italian regions (Apulia, Calabria, Campania, and Tuscany) (Table 1). Target DNA was amplified in a volume of $25 \mu \mathrm{l}$ containing $12.5 \mu \mathrm{l}$ of KAPA HiFi Hot Start Ready Mix (KAPA Biosystems, Wilmington, MA), $0.3 \mu \mathrm{M}$ of each primer, including Illumina adaptors (https://www.illumina.com), and $20 \mathrm{ng}$ of DNA template. Reactions consisted of $3 \mathrm{~min}$ of denaturation at $95^{\circ} \mathrm{C}, 35$ cycles of $30 \mathrm{~s}$ at $98^{\circ} \mathrm{C}, 30 \mathrm{~s}$ at $55^{\circ} \mathrm{C}, 40 \mathrm{~s}$ at $72^{\circ} \mathrm{C}$, and a final extension of $1 \mathrm{~min}$ at $72^{\circ} \mathrm{C}$. Nuclease-free water (QIAGEN) replaced template DNA in negative controls. Amplicons were visualized on gel electrophoresis at $1.2 \%$ agarose and sequenced using the MiSeq system according to the manufacturer's protocol for Nextera XT Index Kit v2 (Illumina Inc., San Diego, CA). Purification of samples was achieved using an Agencourt AMPure XP system (Beckman Coulter Genomics, Brea, CA). The final library was normalized and quantified using a Qubit (Life Technologies, Carlsbad, CA). The MiSeq reagent kit v3 (Illumina) was used for long paired-end reads $(2 \times 300 \mathrm{bp})$ sequencing run. The PhiX control was spiked in the final library to add base diversity.

Raw sequencing data (BioProject PRJNA541130) were first trimmed using PEAR 0.9.10 (Zhang et al. 2014) with a minimum quality score of 20 . Reads with less than 280 bases in length were discarded. Sequences were processed within VSEARCH workflow (Rognes et al. 2016). The de novo chimera checking and dereplication were carried out using the uchime and derep_fulllength algorithms. Unique reads were clustered with a similarity threshold of $100 \%$ and defined as sequence types (STs) (Prigigallo et al. 2016). The number of sequences associated with each cluster (the STs) was calculated and their taxonomy was assigned using a local Venturia spp. ITS database (Supplementary Table S1). A further taxa assignment against the UNITE database (Kõljalg et al. 2013) was performed to investigate the eventual presence of sequences of other fungal genera. STs of $V$. oleaginea representing at least 50 reads were compared to determine the frequency and distribution of polymorphic bases in the ITS1-5.8S-ITS2 region. To this aim, sequences were introduced in MEGA7 (Kumar et al. 2016), aligned with the MUSCLE algorithm, and manually checked (Fig. 1).

Design of $V$. oleaginea-specific primers and probe. STs representative of clusters with at least 50 reads from highthroughput sequencing (HTS) and Sanger sequences obtained in this study were compared with GenBank available sequences of $V$. oleaginea and closely related fungal taxa. All sequences were aligned in MEGA7 (Kumar et al. 2016) and manually checked in order to identify appropriate regions shared by all $V$. oleaginea sequences and to avoid as much as possible other closely related taxa. A TaqMan probe (SpOP CCCTGCCTTGAAAA) and the flanking primers (SpO1F CGCCTGCCGGAATTAGC and 
SpO2R CGATTTGACTCGTTCTCAGACTT) were designed using Primer Express software version 3.0 (Applied Biosystems) to target a fragment of $65 \mathrm{bp}$ in the final part of the ITS1 region (Fig. 1; Table 2). The probe was labeled with FAM at the 5' end, quenched with BHQ1 at the $3^{\prime}$ end, and conjugated with a minor groove binder to increase melting temperature (Kutyavin et al. 2000). The primers and probe were synthetized by Applied Biosystems.

Optimization of amplification conditions and evaluation of primer specificity. Given the difficulty of obtaining pure cultures of $V$. oleaginea, amplification conditions were optimized using DNA extracted directly from infected olive leaves showing typical symptoms of OLS. The optimized 20- $\mu$ l PCR reaction contained $10 \mu \mathrm{l}$ of TaqMan Universal PCR Master Mix (Applied Biosystems), $0.4 \mu \mathrm{M}$ of primers, $0.25 \mu \mathrm{M}$ of probe, and $1 \mu \mathrm{l}(20 \mathrm{ng})$ of extracted DNA. Amplification conditions consisted of an initial step of $50^{\circ} \mathrm{C}$ for $2 \mathrm{~min}$ and $95^{\circ} \mathrm{C}$ for $10 \mathrm{~min}$, followed by 40 cycles of $95^{\circ} \mathrm{C}$ for $15 \mathrm{~s}$ and $57^{\circ} \mathrm{C}$ for $60 \mathrm{~s}$. All reactions were incubated in a StepOnePlus real-time thermocycler (Applied Biosystems) and data were analyzed according to the manufacturer's instructions. The quantification cycle $(\mathrm{Cq})$ values were calculated automatically by determining the PCR cycle number at which the reporter fluorescence exceeded the background.

To evaluate the specificity of the qPCR method, 20 samples of healthy olive leaves were collected in Puglia/Apulia and Calabria from resistant cultivars Leccino and Roggianella (Agosteo and Schena 2011) in areas with no historical record of OLS for at least
3 years. Each sample was collected from an individual tree. Since $V$. oleaginea affects leaves primarily in the lower canopy, all samples were collected from the top of the canopy (Agosteo and Schena 2011). Leaves with typical symptoms of OLS were used as positive controls. DNA was extracted from all leaves as described above and amplified according to optimized amplification conditions. Negative controls, in which water replaced template DNA, were used in all reactions.

Construction of calibration curves for quantitative analyses. A PCR amplicon of $497 \mathrm{bp}$ was obtained using the generic primers Ve1F and Ve2R from symptomatic olive leaves. The amplicon was purified and cloned into a plasmid vector (pGEM-T Easy Vector System I) according to the manufacturer's protocol (Promega Corporation). The plasmid was extracted using the Quantum Prep Plasmid Miniprep Kit (Bio-Rad Laboratories) and quantified using a NanoDrop spectrophotometer (NanoDrop Technologies). Plasmid DNA was serially diluted 10 -fold to yield a final DNA concentration ranging from $100 \mathrm{pg}$ to $10 \mathrm{ag}$ and amplified in triplicate as described above. Water replaced template DNA in negative controls. To evaluate the possible inhibitory effect of DNA extracts from olive tissues, an identical series of amplifications was performed by spiking reaction mixtures with $1 \mu \mathrm{l}$ of DNA from noninfected olive leaves. The StepOnePlus associated software (Applied Biosystems) was utilized to plot DNA quantities against the corresponding $\mathrm{Cq}$ values and generate a calibration curve and corresponding regression equation, coefficient of determination $\left(R^{2}\right)$, and PCR efficiency (Kralik and Ricchi

TABLE 1. Source location of olive leaves with typical symptoms of olive leaf spot (caused by Venturia oleaginea) collected in Italy, Greece, Spain, and France and the corresponding accession number for sequences of the ITS1-5.8S-ITS2 region of the rDNA for these samples

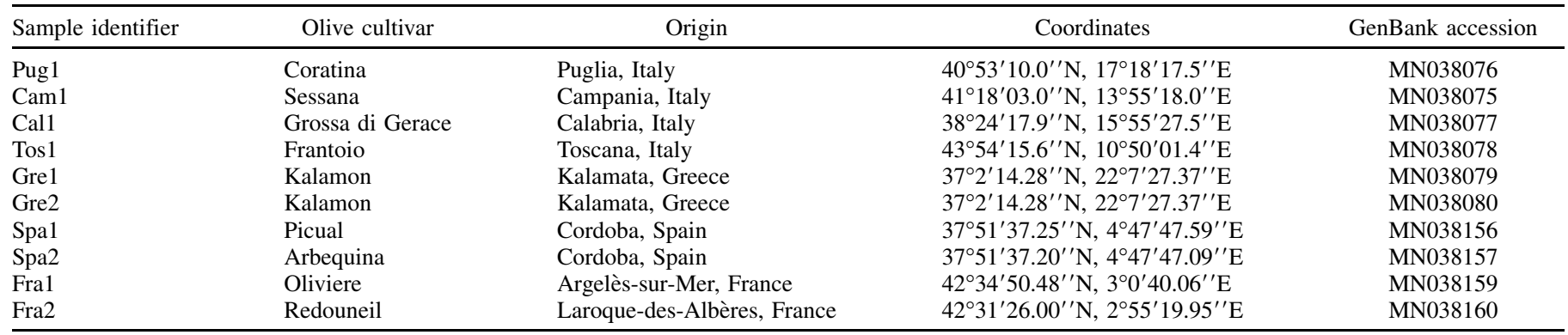

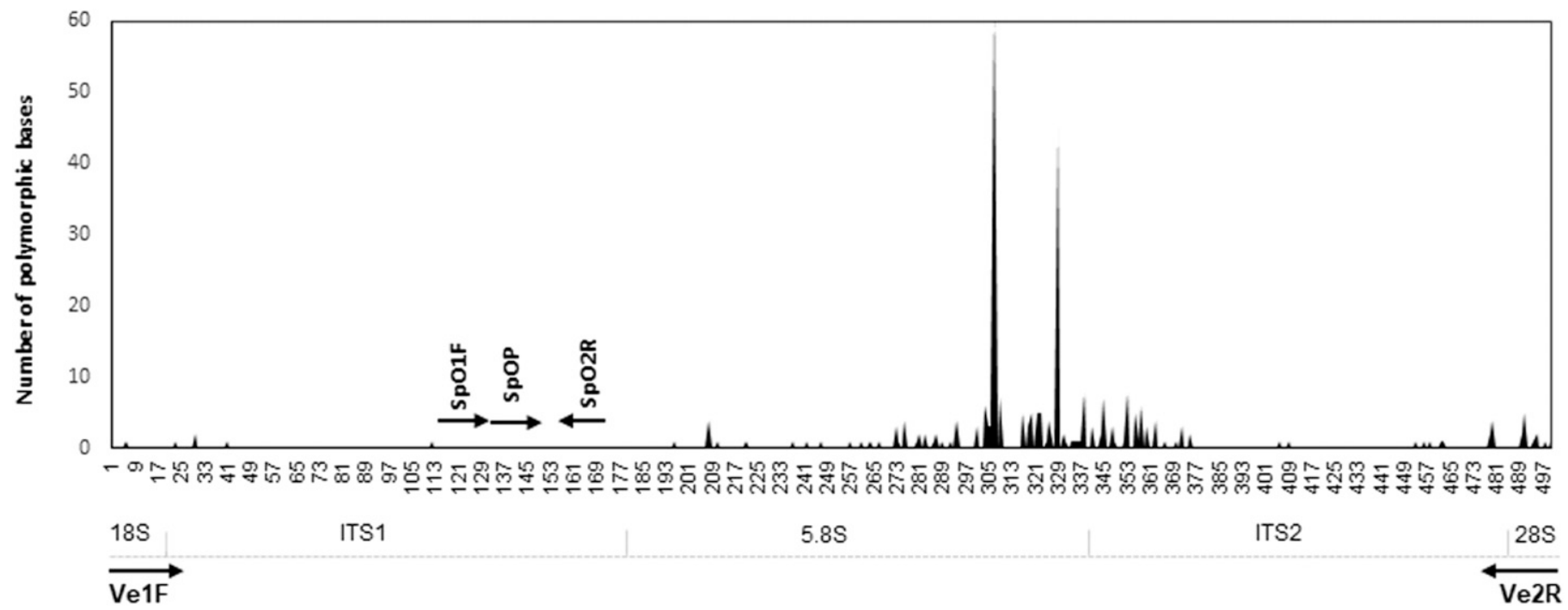

Fig. 1. Distribution of polymorphic bases in the ITS1-5.8S-ITS2 region of the rDNA of Venturia oleaginea based on results from metabarcoding studies on DNA extracted from olive leaves. Analyses were performed using sequence types representative of at least 50 reads $(n=202)$. The location of primers utilized for metabarcoding analyses ( $\mathrm{Ve} 1 \mathrm{~F}$ and $\mathrm{Ve} 2 \mathrm{R}$ ) and primers and probes designed to specifically detect and quantify V. oleaginea (SpO1F, SpOP, and SpO2R) is indicated. 
2017). Finally, DNA quantities were converted to number of copies of target DNA assuming that the average weight of a base pair is $650 \mathrm{Da}$ (http://cels.uri.edu/gsc/cndna.html).

Analysis of latent infections in olive leaves. Samples were taken from two organic olive groves of cultivars Carolea and Grossa di Gerace located in the Calabria region in the municipalities of Lamezia Terme $\left(38^{\circ} 55^{\prime} 25.0^{\prime \prime} \mathrm{N} 16^{\circ} 19^{\prime} 37.0^{\prime \prime} \mathrm{E}\right.$, elevation $\left.65 \mathrm{~m}\right)$ and Locri $\left(38^{\circ} 13^{\prime} 09.0^{\prime \prime} \mathrm{N} 16^{\circ} 14^{\prime} 13.0^{\prime \prime} \mathrm{E}\right.$, elevation $\left.17 \mathrm{~m}\right)$, respectively. Records showed that OLS was historically severe at both sites. Furthermore, they have similar annual average temperatures $\left(17.2\right.$ and $\left.17.9^{\circ} \mathrm{C}\right)$ and rainfall (887 and $824 \mathrm{~mm}$ ), respectively (https://it.climate-data.org/).

At both sites, asymptomatic young shoots were collected every 20 days from March to May 2016 (Table 3). At each sampling time, a total of 30 shoots (three biological replicates of 10 shoots) were collected from a group of three olive trees. Samples were quickly transferred to the laboratory, cut to separate leaves at different growth stages, and lyophilized to perform DNA extractions. According to Sanz-Cortés et al. (2002), stage 0 was associated with developing buds, followed by stage 1 when the first leaves are completely separated; subsequent young leaves observed on the shoot constitute stages 2, 3, and 4, respectively. The size of leaves at each stage was similar in the two groves and increased slightly from sampling time 1 to 4 (Table 3). Because samples were collected during a period of rapid growth, each sample time represented a snapshot with entirely new leaf stages compared with those collected 20 days before.

DNA was amplified in triplicate with the optimized TaqMan PCR assay to detect and quantify $V$. oleaginea. Furthermore, to take into account small variations in sample size and efficiency of extraction and amplification, all samples were amplified with a universal TaqMan system (Schena et al. 2006). Samples of DNA extracted from healthy olive leaves were included as negative controls in all amplification sets.

The average $\mathrm{Cq}$ values obtained for each biological replicate with specific primers were normalized using $\mathrm{Cq}$ values from the universal method as described by Schena et al. (2017). The concentration of $V$. oleaginea DNA in each sample was extrapolated using the corrected $\mathrm{Cq}$ values and the mathematical function of the calibration curve. This concentration was utilized to estimate the DNA copies per gram of lyophilized leaf tissue, considering that qPCR reactions contained $1 \mu \mathrm{l}$ of extracted DNA and that total DNA was extracted from $40 \mathrm{mg}$ of dry leaf tissues and diluted in $50 \mu \mathrm{l}$ of water. The concentrations of the pathogen in the different samples were analyzed using a one-way analysis of variance to compare

TABLE 2. Alignment of the distal sequence of the ITS1 region of the rDNA of Venturia oleaginea (Fig. 1) on which specific TaqMan primers and probes were designed ${ }^{\text {a }}$

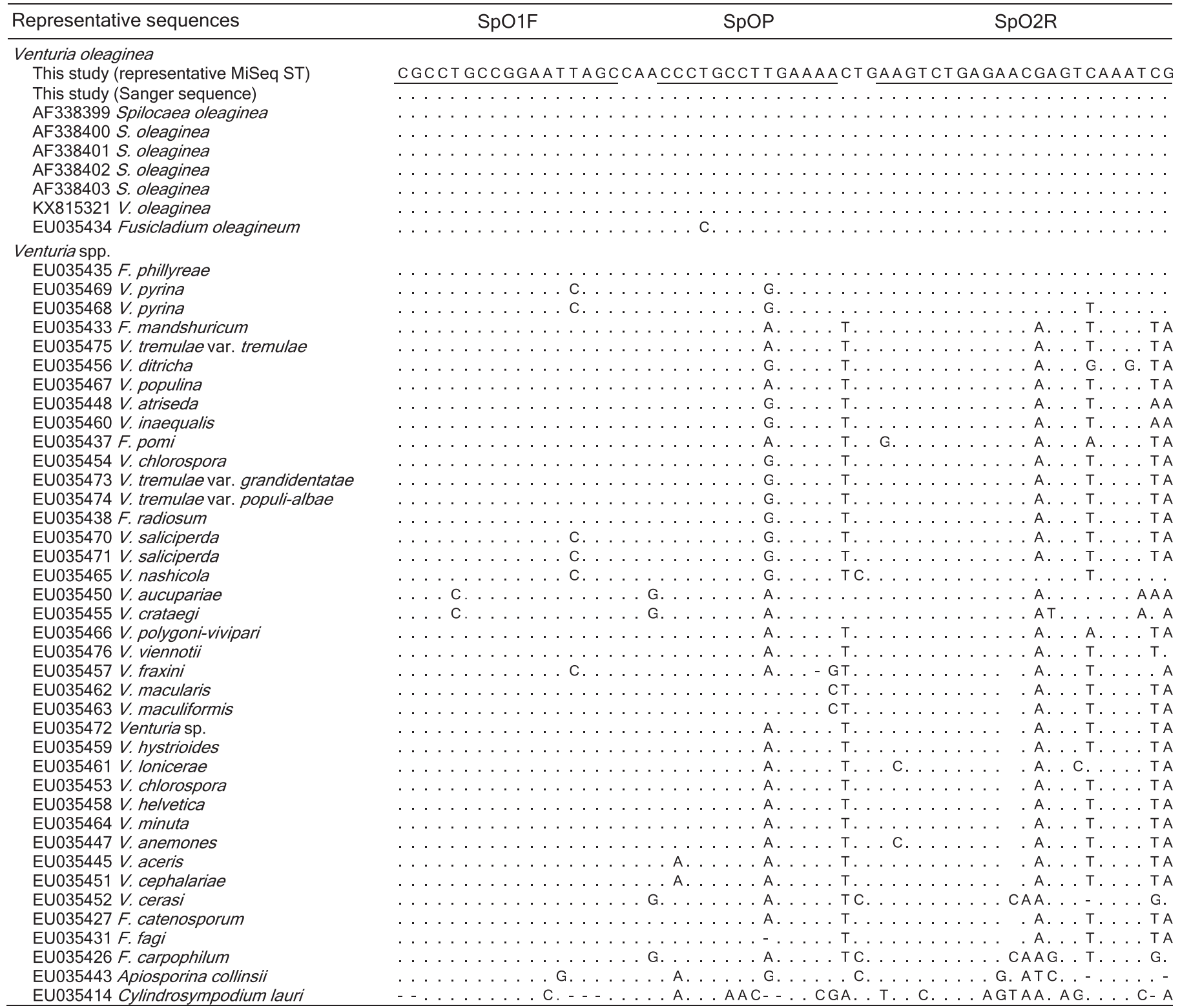

a Alignment comprises a representative sequence type (ST) from metabarcoding analyses, a representative Sanger sequence obtained in this study, all GenBank available sequences of $V$. oleaginea, and sequences of closely related taxa (Crous et al. 2007). 
stages (within each sampling time and across all times) and times (within each stage and across all stages). A post hoc multiple comparison was performed using Tukey's honest significant difference test $(\alpha=0.05)$. IBM SPSS Statistics software (IBM, Milan, Italy) was used for the analysis.

\section{RESULTS}

Amplification and sequencing of the ITS region of V. oleaginea. The modified protocol we utilized to extract nucleic acids from olive leaves yielded DNA of good quality suitable for Sanger and MiSeq sequencing as well as for qPCR analyses. Approximately 4,500 ng of DNA was extracted from each sample with an average concentration of $90 \mathrm{ng} / \mu \mathrm{l}$. The A260/A280 ratio was consistently in the range 1.8 to 1.9 , indicating pure DNA, whereas the A260/A230 ratio was approximately 1, indicating limited contamination with aromatic compounds and/or carbohydrates.

Primers Ve1F and Ve2R consistently enabled the amplification of a PCR fragment of approximately $500 \mathrm{bp}$ from all olive samples showing typical symptoms of OLS. This fragment was pure enough to enable direct Sanger sequencing. Sequences were 497 bp and identical to each other, regardless of source location in Italy, Greece, Spain, or France (Table 1). An identical sequence was also determined for the cloned PCR fragment and for an isolate deposited in GenBank as F. phillyreae (EU035435). On the contrary, few polymorphic bases differentiated sequences obtained in this study from all other sequences deposited in GenBank as F. oleaginum (EU035435), S. oleagina (AF338399-338403), and V. oleaginea (KX815321) (Supplementary Table S1).

HTS analyses. After quality and chimera filtration procedures, the sequencing of DNA from the four leaf samples collected in Apulia, Calabria, Campania, and Tuscany yielded a total of 444,276 reads. The dereplication and clustering of these reads using a similarity threshold of $100 \%$ enabled the identification of 344,918 clusters or STs. There were 202 clusters with $>50$ reads containing a cumulative total of 61,625 sequences. In particular, the most abundant ST was representative of 19,875 reads and its sequence was identical to those determined by Sanger sequencing. Abundant clusters were followed by a swarm of clusters with few reads, mostly differentiated by single polymorphic bases. Almost all read clusters were assigned to the species V. oleaginea. A single exception was a ST, representative of a cluster with 206 reads, assigned to Aureobasidium pullulans.

The analysis of the frequency and distribution of polymorphic bases by comparing STs representative of at least 50 reads showed a low variation within the ITS1 region compared with the second half of the $5.8 \mathrm{~S}$ gene and, to a lesser extent, the first part of the ITS2 region (Fig. 1).

Design of $V$. oleaginea-specific primers and probe. A DNA region suitable for the design of a TaqMan marker (two specific primers and a probe) was identified in the second section of the ITS1 region (Fig. 1). The selected fragment was identical in all MiSeq STs representing clusters of at least 50 reads, in Sanger sequences identified in this study, and in previously deposited sequences (Table 2). A single exception was an isolate of
$V$. oleaginea sequenced in New Zealand (EU035434) that was differentiated from other sequences because of a " $C$ " substituting a "T" in the final part of the ITS1 region (Table 2). On the other hand, with the exception of $F$. phillyreae, the selected fragment of $V$. oleaginea was different compared with homologous regions of all other Fusicladium species to which it was compared (Table 2).

Calibration curves, sensitivity, and specificity. The construction of calibration curves using serial dilutions of a cloned PCR fragment revealed a detection limit of 100 ag corresponding to approximately 26 copies of target DNA and an average Cq value of 35.5 (Fig. 2). The efficiency of the amplification reaction was $101.91 \%$. The resulting linear regression equation $(y=-3.277 x+$ 40.31) related the number of copies of DNA to the $\mathrm{Cq}$ values with a coefficient of determination $\left(R^{2}\right)$ of 0.998 (Fig. 2). The spiking of reaction mixtures with $1 \mu \mathrm{l}$ of DNA from noninfected olive leaves slightly reduced the fluorescence signal but did not have any impact on the detection limit, regression parameters, or $R^{2}$ value.

The specificity of primers and probe was confirmed by screening healthy olive leaves. None of the samples tested produced a significant increase in fluorescence. On the contrary, the presence of the pathogen was confirmed in all positive controls (leaves showing typical symptoms of OLS) with an average Cq value of 23.3.

Analysis of olive leaves at different growth stages and timings. In concordance with the calibration curve, the detection limit of the pathogen on naturally infected leaves was established at an average $\mathrm{Cq}$ value $\leq 35.5$. According to this criterion, all leaf stages and times tested from both the Lamezia Terme and Locri localities were found to be infected since all biological replicates had a $\mathrm{Cq}$ value $\leq 35.5$, although in two samples, only two of three technical replicates had a $\mathrm{Cq}$ value $\leq 35.5$. For these two latter samples, the concentration of the pathogen was estimated using $\mathrm{Cq}$ values from the two positive technical replicates.

In samples collected in Lamezia Terme, the highest concentration of pathogen was determined on 20 March 2016 (T2), with 10.4,

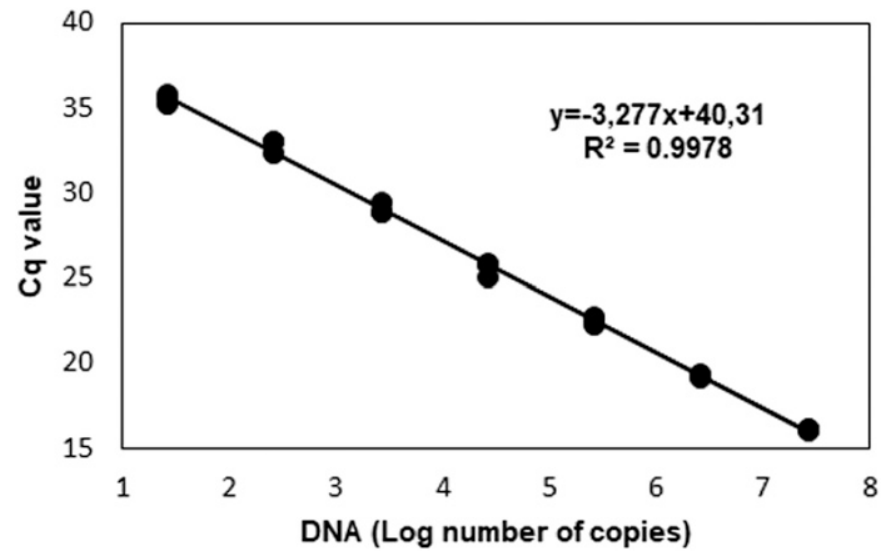

Fig. 2. The calibration curve, regression equation, and coefficient of determination for the quantitative PCR method utilized to detect and quantify Venturia oleaginea in olive leaves. The curve was generated using serial dilutions of a cloned fragment of the ITS1-5.8S-ITS2 regions (Fig. 1) spiked with DNA from noninfected olive leaves. $\mathrm{Cq}=$ quantification cycle.

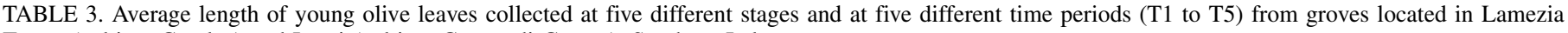
Terme (cultivar Carolea) and Locri (cultivar Grossa di Gerace), Southern Italy ${ }^{\mathrm{a}}$

\begin{tabular}{|c|c|c|c|c|c|c|c|c|c|c|}
\hline \multirow[b]{2}{*}{ Sampling time } & \multicolumn{2}{|c|}{ Stage 0} & \multicolumn{2}{|c|}{ Stage 1} & \multicolumn{2}{|c|}{ Stage 2} & \multicolumn{2}{|c|}{ Stage 3} & \multicolumn{2}{|c|}{ Stage 4} \\
\hline & Lamezia & Locri & Lamezia & Locri & Lamezia & Locri & Lamezia & Locri & Lamezia & Locri \\
\hline T1 (2 March 2016) & $5.4 \pm 0.68$ & NA & $12 \pm 1.11$ & NA & $21 \pm 1.58$ & NA & NA & NA & NA & NA \\
\hline T2 (20 March 2016) & $7.7 \pm 1.78$ & $7.9 \pm 1.32$ & $18.8 \pm 2.91$ & $19.4 \pm 2.99$ & $24 \pm 3.22$ & $24.3 \pm 2.45$ & NA & $27.6 \pm 5.57$ & NA & NA \\
\hline T3 (6 April 2016) & $10.0 \pm 0.89$ & $10.2 \pm 0.81$ & $19.4 \pm 0.41$ & $19.5 \pm 0.38$ & $30.4 \pm 3.79$ & $30.3 \pm 3.29$ & $38 \pm 1.54$ & $38.3 \pm 1.63$ & $52.9 \pm 2.78$ & $53.4 \pm 3.52$ \\
\hline T4 (22 April 2016) & $13.4 \pm 0.41$ & $13.5 \pm 0.48$ & $28.8 \pm 0.82$ & $29 \pm 0.56$ & $44.1 \pm 1.11$ & $44.5 \pm 0.98$ & $64.2 \pm 6.46$ & $63.7 \pm 8.98$ & $89.5 \pm 0.89$ & $89.1 \pm 1.22$ \\
\hline T5 (13 May 2016) & $14.6 \pm 2.8$ & $14.5 \pm 1.94$ & $26.7 \pm 2.89$ & $26.5 \pm 1.97$ & $44 \pm 0.81$ & $44.2 \pm 1.06$ & $54.7 \pm 1.13$ & $55.8 \pm 1.63$ & $65.5 \pm 4.87$ & $64.3 \pm 6.36$ \\
\hline
\end{tabular}

a Data are presented as the average length \pm standard error (in millimeters). NA = not analyzed samples. 
16.7, and 19.1 million of copies of DNA per gram of dry tissue at leaves of stages 0,1 and 2, respectively (Fig. 3). Significantly lower quantities of the pathogen were determined on 2 March 2016 (T1) and 6 April 2016 (T3), with 3.6 to 6.0 (T1) and 0.69 to 4.5 (T3) million copies, respectively. The pathogen titer further decreased on 22 April 2016 (T4) and 13 May 2016 (T5) (Fig. 3).

Similar to the samples from Lamezia Terme, the samples collected in Locri revealed a significantly higher concentration of the pathogen in leaves collected at $\mathrm{T} 2$ followed by those collected at T3 (Fig. 4). Compared with T2 and T3, significantly lower concentrations were detected at $\mathrm{T} 4$ and $\mathrm{T} 5$, while $\mathrm{T} 1$ was not analyzed. Overall, a more gradual reduction of the pathogen concentration over time was observed at Locri compared with Lamezia Terme (Figs. 3 and 4).

At both localities, the pathogen was detected at all growth stages, but the comparison of the DNA concentration among the four growth stages ( 0 to 4 ) within each sampling time (T1 to T5) over the sampling periods investigated did not show any significant difference (Figs. 3 and 4).

\section{DISCUSSION}

Fundamental prerequisites for an effective and reliable PCR-based molecular detection method are specificity and the absence of crossamplification from related taxa, while detecting all possible genetic variants of the target species. These conditions are generally satisfied by preliminary in silico analyses and, more importantly, assessing specificity against a wide range of representative isolates from target and related species (Schena et al. 2013). In the case of difficult-toculture biotrophic fungi like V. oleaginea, the evaluation of specificity and the ability to detect variants of the target species are challenged by the lack of pure in vitro cultures. To overcome this limitation, we compared the few available ITS sequences of the pathogen with those of related species in order to design new primers (Ve1F and Ve2R) enabling the amplification and sequencing of the entire $V$. oleaginea ITS1-5.8S-ITS2 region directly from infected olive leaves. These primers were intentionally designed to target quite conserved regions shared by several Fusicladium-related species (the final region of the $18 \mathrm{~S}$ gene and boundary between the ITS2 and $28 \mathrm{~S}$ gene) in order to cover possible ITS variants of $V$. oleaginea and simultaneously avoid unrelated fungal species.

Sanger sequencing of $V$. oleaginea fragments amplified directly from leaves with primers $\mathrm{Ve} 1 \mathrm{~F}$ and $\mathrm{Ve} 2 \mathrm{R}$ yielded identical sequences for all of the samples from Italy, France, Greece, and Spain. This sequence was also identical to the most abundant ST obtained by MiSeq analysis but did not perfectly match any of the previously deposited sequences of five isolates from Spain (AF338399-403), one from New Zealand (EU035434), and one of unknown origin (KX815321) (Supplementary Table S1). Since all previously deposited sequences also differed from each other by at least one nucleotide, further investigation may be worthwhile. Nevertheless, we purposely designed specific qPCR primers and probes targeting a fragment of the ITS1 region shared by most sequences in order to guarantee the capability of our method in covering possible ITS variants. Only the New Zealand isolate (CBS113427) showed the presence of a polymorphic base in the target region of the probe (Table 2). Further work is needed to confirm the existence of this polymorphic base and its geographic distribution.

Unlike Sanger sequencing, HTS analyses produced a high number of STs for $V$. oleaginea, mostly differentiated by single nucleotides. In agreement with previous reports, these findings may be, at least partially, the result of ITS intraspecies and/or intraisolate variability attributable to the multicopy nature of

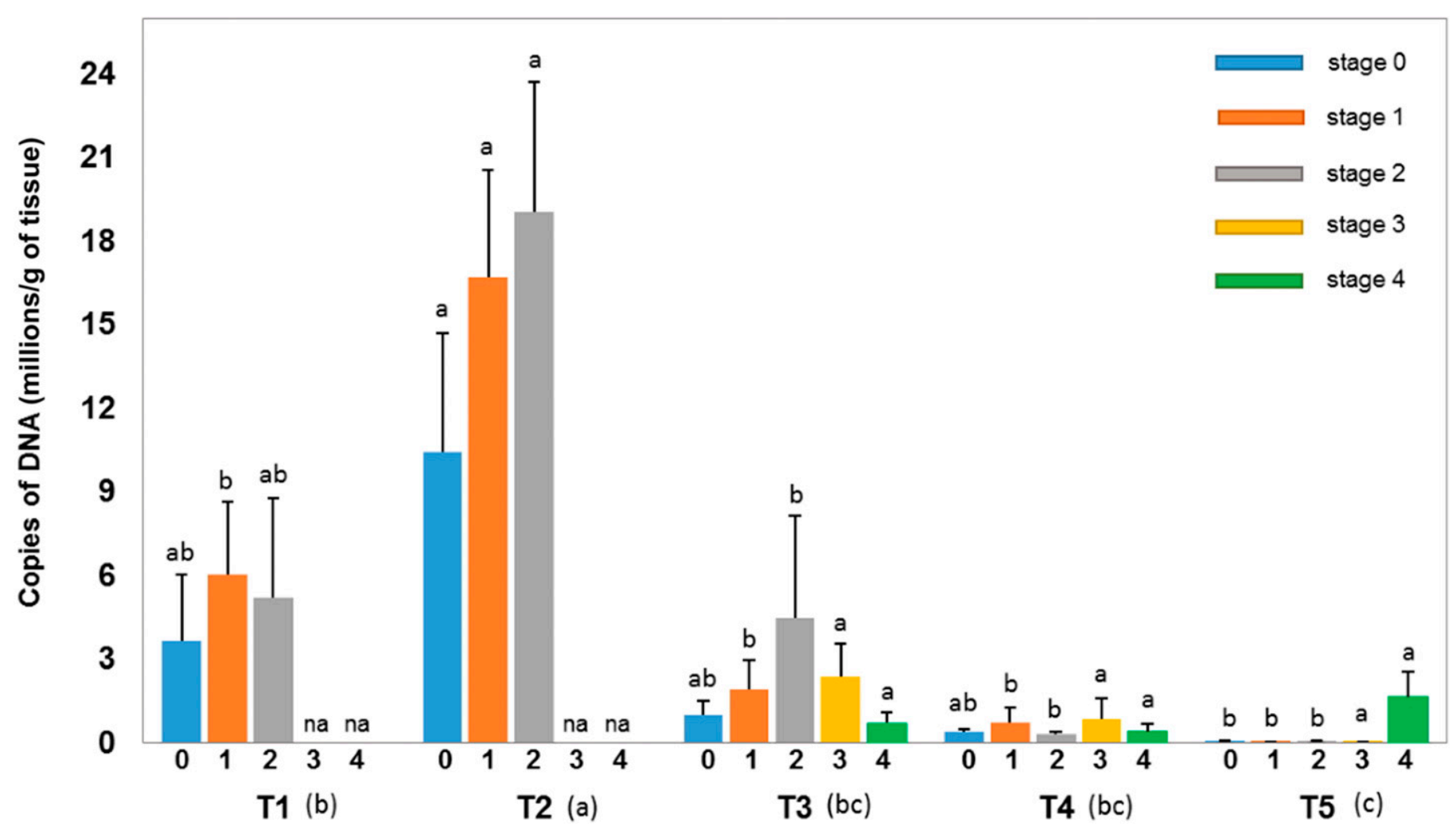

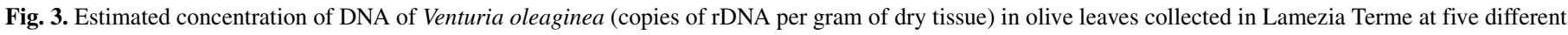

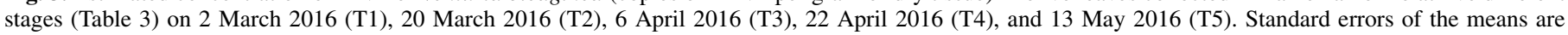

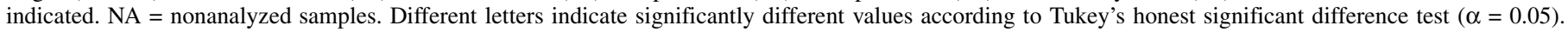

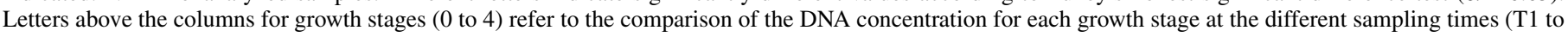

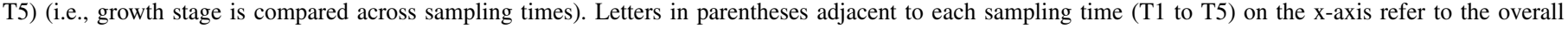
comparisons between sampling times. No significant differences were observed among stages (data not shown). 
rDNA (Zhao et al. 2015). Since the most abundant ST was identical to the Sanger sequences, it is possible that less abundant variants were not observed in Sanger sequencing simply because of their low frequency. However, it is important to point out that MiSeq data are inappropriate to determine the actual presence and incidence of polymorphic bases as a result of errors typically introduced by next-generation sequencing approaches (Pfeiffer et al. 2018). The observed prevalence of polymorphic bases in the median part of the sequenced region indirectly suggests that most observed sequence variations are attributable to sequencing errors. In fact, we used a long paired-end reads $(2 \times 300 \mathrm{bp})$ approach to analyze fragments of approximately $500 \mathrm{bp}$, and substitution errors in Illumina MiSeq platform are known to increase toward the end of the read (Schirmer et al. 2015). Furthermore, the observed higher incidence of variation within the conserved 5.8S gene compared with variable ITS1 and ITS2 regions corroborates the likely role of sequencing errors.

Regardless of sequencing shortcomings, MiSeq data were valuable to confirm the absence of other fungal species phylogenetically related to $V$. oleaginea in the olive phyllosphere. In fact, reads were almost all identified as $V$. oleaginea with a single ST associated with the distant species $A$. pullulans. A single exception was represented by $F$. phillyrea that shares identical ITS sequences with $V$. oleaginea, despite this species being strictly associated with the plant genus Phillyrea (Schubert et al. 2003). From a practical point of view, the absence of other Venturia species in the olive phyllosphere is important to exclude the risk of specificity issues in qPCR analyses. A minimum of two to three unique bases is considered necessary to generate highly specific primers and probes (Schena et al. 2013) and this condition was not always satisfied in this study owing to few closely related species with very similar ITS sequences (Table 2). Nevertheless, the specificity of the qPCR method we developed was experimentally confirmed by the absence of amplification with all healthy olive leaves used as negative controls.

The qPCR method was not influenced by olive DNA. Furthermore, the extracted DNA was of good quality and enabled the detection of $V$. oleaginea DNA over a wide range of concentrations. The detection limit was established at approximately $100 \mathrm{ag}$, corresponding to an average $\mathrm{Cq}$ value of 35.5 and to approximately 26 copies of target DNA. Considering the multicopy nature of the rDNA, this detection limit suggests that very few, or even a single, pathogen cells could be detected in $1 \mathrm{~g}$ of leaf tissue. In this context, the selection of the ITS1 region as the target gene was useful to increase the sensitivity of the method. However, it is also important to point out that the multicopy nature rDNA complicates quantitative analyses since the exact number of rDNA repeats is unknown in $V$. oleaginea and the number of copies may vary within fungal species. For instance, Bilodeau et al. (2012) estimated that copies of rDNA in Verticillium dahliae can range from approximately 24 to 73 per haploid genome. Furthermore, a consistent part of total DNA can be lost during extractions, resulting in underestimation of the pathogen concentration (Gusberti et al. 2012; Luo et al. 2009). Despite these shortcomings, we contend the potential error is of secondary importance since the high sensitivity of the method enabled the quantification of very low infection titers, which might correspond to the amount present at the onset of infection or during latent, nonsymptomatic infections (Sanzani et al. 2014).

In this study, the use of the qPCR method enabled detection of $V$. oleaginea in very young olive leaves at a phenological phase

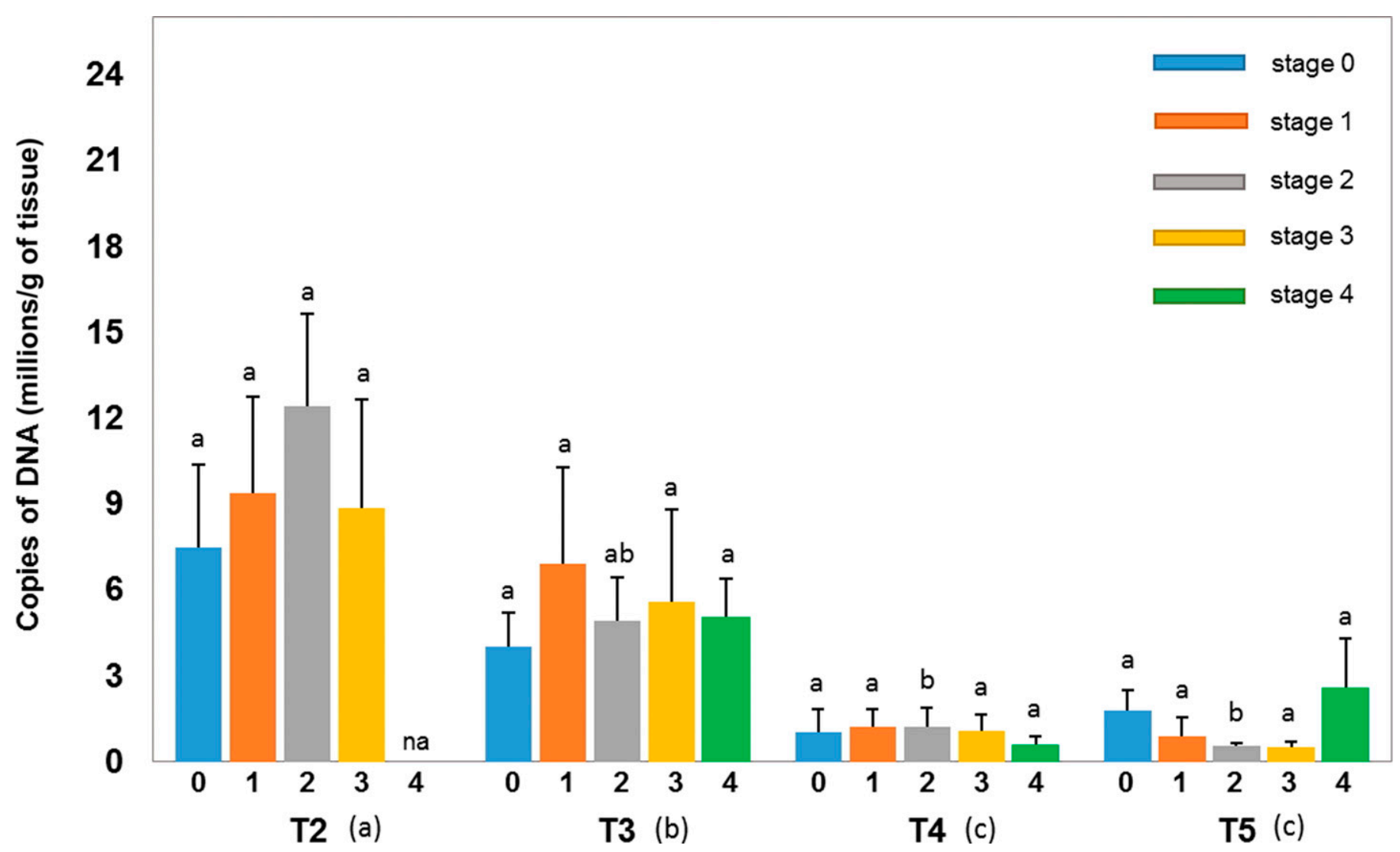

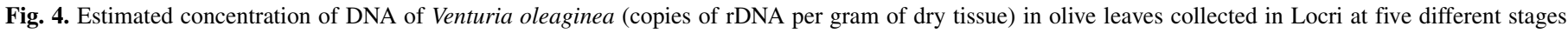

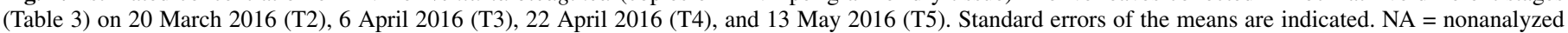

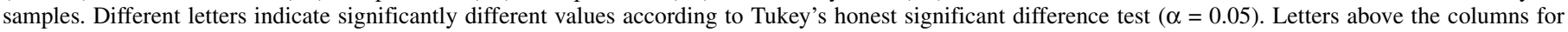

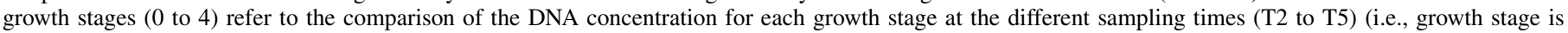

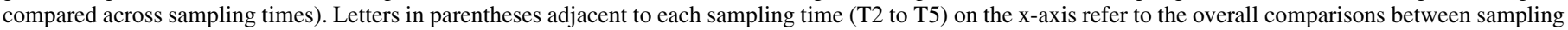
times. No significant differences were observed among stages (data not shown). 
that had not previously been diagnosed because of a lack of appropriate methods. The $\mathrm{NaOH}$ assay is widely used to detect the percentage of infected leaves in the summer, in order to schedule the autumnal fungicide treatments. However, the method is not applicable to the young, small and tender leaves in early spring, because tissues can be totally degraded or show spots unrelated to the presence of the pathogen (false positives). Furthermore, the $\mathrm{NaOH}$ assay diagnosis on asymptomatic leaves seems to be possible only after at least 10 days since infection (Loprieno and Tenerini 1959). In spring, after a rainy period, the rapid increase in hot and dry conditions may halt the growth of the pathogen after infection, precluding symptom development at that time. Under these conditions, the $\mathrm{NaOH}$ assay cannot detect the pathogen as a result of the latency, and this results in an underestimation of the disease.

Analyses conducted at two different localities (Locri and Lamezia Terme) unexpectedly revealed the presence of the pathogen in asymptomatic leaves from the very early stages of growth (leaves with a mean length of $5.4 \mathrm{~mm}$ ). Furthermore, the concentration of the DNA did not change significantly with the growth stage, suggesting that foliage was highly susceptible to infection and colonization by $V$. oleaginea from early leaf development stages. These results are novel in regard to OLS because young leaves of the current year have in the past been considered immune or at least less sensitive to infection (Peglion 1928). Furthermore, these observations are supported by similar results from a related pathosystem in which young leaves of pecan are highly susceptible to the scab pathogen, V. effusa (Gottwald 1985; Turechek and Stevenson 1998). From a practical point of view, our findings are particularly important for improved management of OLS. Spring fungicide treatments are considered the most important for the control of OLS but are commonly scheduled when the vegetative growth is about two-thirds of the final expected development. Our findings suggest the need for earlier fungicide treatments because the traditional schedules are inappropriate to prevent new infections. This is particularly true for preventive fungicides like cupric salts, which are the most commonly utilized in integrated pest management (IPM) programs on olive to control OLS, and are the only chemicals permitted in organic farming.

Regardless of the growth stage, the samples from Lamezia Terme showed a higher concentration of pathogen DNA at T2 (20 March 2016) compared with T1 (2 March 2016), followed by a significant decrease in the quantity of the pathogen at the following sampling times. A similar trend was revealed in Locri, even though the first sampling time (T1) was not included. Although further investigations are needed to confirm this trend, the development of the disease is likely the result of environmental factors. From T1 to T2 (beginning of March), new infections increase progressively because moisture is not a limiting factor and temperatures become milder and suitable for infection and colonization of the host. Subsequently, temperature is no longer a limiting factor, but lower humidity and rainfall progressively result in conditions less suitable for new infections as the season proceeds and the proliferation and spore production of the pathogen declines inside the tissues.

In conclusion, a new molecular detection method based on qPCR was developed to detect and quantify DNA of $V$. oleaginea in olive tissues. Use of the method provided important insights into the infection of young olive leaves, which has practical implications for optimizing management of OLS, including through IPM strategies. The new detection method represents a valuable instrument for future investigations and will contribute significantly to advancing our knowledge on the biology and epidemiology of V. oleaginea. The diagnostic is specific, enables quantification of the pathogen, and can be applied to all growth stages of the leaves. Although we focused our investigations on leaves, the method can be easily implemented to detect $V$. oleaginea in other olive organs including drupes, fruit peduncles, leaf petioles, and inflorescences, which frequently show symptoms that are not easily recognizable and cannot be analyzed using the traditional $\mathrm{NaOH}$ assay.

\section{ACKNOWLEDGMENTS}

We thank Antonios Zambounis (Institute of Plant Breeding and Genetic Resources, Greece), Carlos Josè Lopez Herrera (Institute for Sustainable Agriculture, Spain), David Ruano-Rosa (Agricultural Technological Institute of Castilla and Leon, Spain), Margaux Allix (CIVAM BIO 66 Association, Perpignan, France), and Willy Couanon (French Technical Centre for Olive Production) for providing OLS symptomatic olive leaves. We also thank Ann Davies for the revision of the English text.

\section{LITERATURE CITED}

Agosteo, G. E., and Schena, L. 2011. Olive leaf spot. Pages 143-176 in: Olive Diseases and Disorders. Transworld Research Network, Trivandrum, India.

Bilodeau, G. J., Koike, S. T., Uribe, P., and Martin, F. N. 2012. Development of an assay for rapid detection and quantification of Verticillium dahliae in soil. Phytopathology 102:331-343.

Crous, P. W., Schubert, K., Braun, U., de Hoog, G. S., Hocking, A. D., Shin, H. D., et al. 2007. Opportunistic, human-pathogenic species in the Herpotrichiellaceae are phenotypically similar to saprobic or phytopathogenic species in the Venturiaceae. Stud. Mycol. 58:185-217.

Doyle, J., and Doyle, J. 1987. A rapid DNA isolation procedure from small quantities of fresh leaf tissues. Phytochem. Bull. 19:11-15.

González-Lamothe, R., Segura, R., Trapero, A., Baldoni, L., Botella, M. A., and Valpuesta, V. 2002. Phylogeny of the fungus Spilocaea oleagina, the causal agent of peacock leaf spot in olive. FEMS Microbiol. Lett. 210:149-155.

Gottwald, T. R. 1985. Influence of temperature, leaf wetness period, leaf age, and spore concentration on infection of pecan leaves by conidia of Cladosporium caryigenum. Phytopathology 75:190-194.

Graniti, A. 1993. Olive scab: A review. EPPO Bull. 23:377-384.

Gusberti, M., Patocchi, A., Gessler, C., and Broggini, G. A. L. 2012. Quantification of Venturia inaequalis growth in Malus $\times$ domestica with quantitative real-time polymerase chain reaction. Plant Dis. 96:1791-1797.

Kõljalg, U., Nilsson, R. H., Abarenkov, K., Tedersoo, L., Taylor, A. F. S., Bahram, M., et al. 2013. Towards a unified paradigm for sequence-based identification of fungi. Mol. Ecol. 22:5271-5277.

Kralik, P., and Ricchi, M. 2017. A basic guide to real time PCR in microbial diagnostics: Definitions, parameters, and everything. Front. Microbiol. 8:108.

Kumar, S., Stecher, G., and Tamura, K. 2016. MEGA7: Molecular Evolutionary Genetics Analysis version 7.0 for bigger datasets. Mol. Biol. Evol. 33:1870-1874.

Kutyavin, I. V., Afonina, I. A., Mills, A., Gorn, V. V., Lukhtanov, E. A., Belousov, E. S., et al. 2000. 3'-Minor groove binder-DNA probes increase sequence specificity at PCR extension temperatures. Nucleic Acids Res. 28:655-661.

Lanza, B., Ragnelli, A. M., Priore, M., and Aimola, P. 2017. Morphological and histochemical investigation of the response of Olea europaea leaves to fungal attack by Spilocaea oleagina. Plant Pathol. 66:1239-1247.

Loprieno, N., and Tenerini, I. 1959. "Metodo per la diagnosi precoce dell'occhio di pavone dell'olivo (Cycloconium oleaginum Cast.)". J. Phytopathol. 34:385-392.

Luo, Y., Gao, W., Doster, M., and Michailides, T. J. 2009. Quantification of conidial density of Aspergillus flavus and A. parasiticus in soil from almond orchards using real-time PCR. J. Appl. Microbiol. 106:1649-1660.

Peglion, V. 1928. Vaiuolo od Occhio di pavone. Pages 628-634 in: Le malattie crittogamiche delle piante cultivate. Casa Editrice Fratelli Ottavi, Casale Monferrato, Italy.

Pfeiffer, F., Gröber, C., Blank, M., Händler, K., Beyer, M., Schultze, J. L., et al. 2018. Systematic evaluation of error rates and causes in short samples in next-generation sequencing. Sci. Rep. 8:10950.

Prigigallo, M. I., Abdelfattah, A., Cacciola, S. O., Faedda, R., Sanzani, S. M., Cooke, D. E. L., and Schena, L. 2016. Metabarcoding analysis of Phytophthora diversity using genus-specific primers and 454 pyrosequencing. Phytopathology 106:305-313.

Rognes, T., Flouri, T., Nichols, B., Quince, C., and Mahé, F. 2016. VSEARCH: A versatile open source tool for metagenomics. PeerJ 4:e2584.

Sanz-Cortés, F., Martinez-Calvo, J., Badenes, M. L., Bleiholder, H., Hack, H., Llacer, G., et al. 2002. Phenological growth stages of olive trees (Olea europaea). Ann. Appl. Biol. 140:151-157.

Sanzani, S. M., Li Destri Nicosia, M. G., Faedda, R., Cacciola, S. O., and Schena, L. 2014. Use of quantitative PCR detection methods to study biocontrol agents and phytopathogenic fungi and oomycetes in environmental samples. J. Phytopathol. 162:1-13.

Schena, L., Abdelfattah, A., Mosca, S., Li Destri Nicosia, M. G., Agosteo, G., and Cacciola, S. O. 2017. Quantitative detection of Colletotrichum godetiae and $C$. acutatum sensu stricto in the phyllosphere and carposphere of olive during four phenological phases. Eur. J. Plant Pathol. 149:337-347.

Schena, L., Hughes, K. J. D., and Cooke, D. E. L. 2006. Detection and quantification of Phytophthora ramorum, $P$. kernoviae, $P$. citricola and $P$. quercina in symptomatic leaves by multiplex real-time PCR. Mol. Plant Pathol. 7:365-379. 
Schena, L., Li Destri Nicosia, M. G., Sanzani, S. M., Faedda, R., Ippolito, A., and Cacciola, S. O. 2013. Development of quantitative PCR detection methods for phytopathogenic fungi and oomycetes. J. Plant Pathol. 95:7-24.

Schena, L., Nigro, F., Ippolito, A., and Gallitelli, D. 2004. Real-time quantitative PCR: A new technology to detect and study phytopathogenic and antagonistic fungi. Eur. J. Plant Pathol. 110:893-908.

Schirmer, M., Ijaz, U. Z., D’ Amore, R., Hall, N., Sloan, W. T., and Quince, C. 2015. Insight into biases and sequencing errors for amplicon sequencing with the Illumina MiSeq platform. Nucleic Acids Res. 43:e37.

Schubert, K., Ristschel, A., and Braun, U. 2003. A monograph of Fusicladium s.lat. (Hyphomycetes). Schlechtendalia (Halle) 9:1-132.

Turechek, W. W., and Stevenson, K. L. 1998. Effects of host resistance, temperature, leaf wetness, and leaf age on infection and lesion development of pecan scab. Phytopathology 88:1294-1301.
Viruega, J. R., Moral, J., Roca, L. F., Navarro, N., and Trapero, A. 2013. Spilocaea oleagina in olive groves of southern Spain: Survival, inoculum production, and dispersal. Plant Dis. 97:1549-1556.

Viruega, J. R., and Trapero-Casas, A. 2002. Effect of temperature, wetness duration and leaf age on infection and development of olive leaf spot. Acta Hortic. 586:797-800.

Wilson, E., and Miller, H. N. 1949. Olive leaf spot and its control with fungicides. Hilgardia 19:1-24.

Zhang, J., Kobert, K., Flouri, T., and Stamatakis, A. 2014. PEAR: A fast and accurate Illumina Paired-End reAd mergeR. Bioinformatics 30:614-620.

Zhao, Y., Tsang, C.-C., Xiao, M., Cheng, J., Xu, Y., Lau, K. S., et al. 2015. Intra-genomic internal transcribed spacer region sequence heterogeneity and molecular diagnosis in clinical microbiology. Int. J. Mol. Sci. 16: $25067-25079$ 